TENDENCIAS

Revista de la Facultad de Ciencias

Económicas y Administrativas.

Universidad de Nariño

ISSN-E 2539-0554

Vol. XXII No. 2 - 2do Semestre 2021

Julio-Diciembre - Páginas 107-129

\title{
EXPOSICIÓN DE MARCA PERSONAL POR MEDIO DE REDES SOCIALES
}

EXPOSURE OF PERSONAL BRAND THROUGH SOCIAL MEDIA

EXPOSIÇÃO DE MARCA PESSOAL ATRAVÉS DA MÍDIA SOCIAL

\author{
Luis Enrique David Tenorio
}

Magíster en Mercadeo, Universidad de Manizales. Docente Facultad de Ciencias Económicas y de la Administración, I.U. Escuela Nacional del Deporte. ORCiD: 0000-0002-5326-7353. E-mail: luis.david@endeporte.edu.co, Colombia.

Recibido: 16 de abril de 2021

Aprobado: 10 de junio de 2021

DOI: https://doi.org/10.22267/rtend.212202.170

\section{Resumen}

La exposición de marca constituye un instrumento de proyección de gran impacto, en la actualidad el uso de medios sociales se convierte en una gran herramienta para lo anterior, utilizado por marcas personales. El conectar personas con seguidores como uno de sus grandes logros, facilitando el alcance de sus objetivos como marca. La presente investigación tuvo como objetivo identificar los factores que facilitan la exposición de una marca personal a través del uso de redes sociales y su posterior uso como medio publicitario. La metodología utilizada se basa en un enfoque cualitativo y como instrumento de recolección de datos se utilizó una entrevista semiestructurada de doce preguntas basadas en los aportes de los diferentes autores especializados en la gestión de marcas corporativas. Con lo anterior, se generó un acercamiento entre la teoría y la práctica a partir de identificar la equivalencia entre los conceptos teóricos y los testimonios de los participantes. Como principal hallazgo se identificó que el sello personal a partir de la 
originalidad es el factor de mayor impulso para aumentar la exposición de una marca personal en redes sociales.

Palabras clave: comunicación; imagen de la marca; marketing; medios sociales; publicidad. JEL: M3; M15; M31; M37; M39

\begin{abstract}
The brand exposure constitutes a projection instrument of great impact, at present the use of social media becomes a great tool for the above, used by personal brands. Connecting people with followers is one of its great achievements, facilitating the achievement of its objectives as a brand. The objective of this research was to identify the factors that facilitate the exposure of a personal brand through the use of social networks and its subsequent use as an advertising medium. The methodology used is based on a qualitative approach and a semi-structured interview of twelve questions was used as a data collection instrument based on the contributions of the different authors specialized in corporate brand management. With the above, a rapprochement between theory and practice was generated by identifying the equivalence between the theoretical concepts and the testimonies of the participants. As the main finding, it was identified that the personal stamp from originality is the factor that drives the greatest drive to increase the exposure of a personal brand on social networks.
\end{abstract}

Keywords: communication; brand image; marketing; social media; advertising. JEL: M3; M15; M31; M37; M39

\title{
Resumo
}

A exposição da marca constitui um instrumento de projeção de grande impacto, atualmente a utilização das redes sociais torna-se uma grande ferramenta para o exposto, utilizada pelas marcas pessoais. Conectar pessoas com seguidores é uma de suas grandes conquistas, facilitando o alcance de seus objetivos como marca. O objetivo desta pesquisa foi identificar os fatores que facilitam a exposição de uma marca pessoal por meio da utilização de redes sociais e sua posterior utilização como meio publicitário. A metodologia utilizada baseia-se na abordagem qualitativa e utilizou-se como instrumento de coleta de dados uma entrevista semiestruturada de doze questões a partir das contribuições de diversos autores especializados em gestão de marcas corporativas. Com o 
exposto, gerou-se uma aproximação entre teoria e prática, identificando a equivalência entre os conceitos teóricos e os depoimentos dos participantes. Como principal achado, identificou-se que a marca pessoal da originalidade é o fator que mais impulsiona o aumento da exposição de uma marca pessoal nas redes sociais.

Palavras-chave: comunicação; imagem de marca; marketing; mídia social; propaganda.

JEL: M3; M15; M31; M37; M39

\section{Introducción}

Las redes sociales se pueden considerar como una estructura social compuesta por individuos conectados por enlaces, lo cuales pueden ser amistad o parentesco, esta forma de relación puede ser tanto individual como global (Iribarren y Moro, 2011), potencializadas por su presencia en la web constituyen un medio de comunicación de crecimiento exponencial, su uso a través de aplicaciones virtuales facilita la interaccion y relación de estos (Gonçalves et al., 2016) conviertiendose un gran medio para la exposición de una marca personal, a partir de lo anterior éstas logran convertirse en un medio de marketing de fácil uso y gran impacto, la difusión de productos a través de estas influenciando de manera particular en la compra de un producto (Peres et al., 2010).

La marca personal constituye una forma de comunicar lo que nos hace diferentes y especiales (Arques, 2012), cómicos, actores, publicistas, cantantes, escritores, etc., con mayor frecuencia utilizan las redes sociales con la finalidad de mostrar su marca personal y de esta forma obtener seguidores, donde la reputación, la seriedad, los temas de interés, la confianza, la coherencia y la persistencia son elementos esenciales para mantenerse, sostenerse y crecer a través de este medio.

Las marcas personales en redes sociales se encuentran en crecimiento y cada vez son más utilizadas como medios de comunicación, permitiendo a productos y servicios apropiarse de este crecimiento para sus necesidades de marketing, el desarrollo de videos para la internet puede convertirse en el medio ideal para la construcción de un marca, algo que no es fácil de obtener pero en el momento que se logra genera aumentar la exposición de esta a un costo ínfimo comprado con los medios tradicionales (Aaker, 2013). 
La importancia de la exposición de una marca a partir del desarrollo de contenidos y como ésta aporta a la construcción de la misma, siendo un fenómeno que deba estudiarse más a profundidad.

Teniendo en cuenta que la gestión de una marca personal, en algunos aspectos, presenta similitudes a una marca corporativa, es necesario analizar este fenómeno, observar el proceso de las mismas y qué se puede aprender, el concepto se remonta básicamente al registro civil del nombre como persona y no desde el branding, haciendo necesario el análisis de esta desde una perspectiva del marketing (Hoyos, 2016). Teniendo en cuenta que se alimenta de los atributos de la persona y por lo que se reconoce y diferencia (Alonso, 2014), generando similitudes en su gestión, a una marca corporativa.

La necesidad de estudiar el creciente fenómeno de marcas personales, parte desde el dinamismo que tiene el mercadeo, el cual siempre busca adaptarse a los nuevos cambios de la sociedad. Esta investigación parte de la necesidad de identificar como una persona puede utilizar las redes sociales para la exposición de su marca y convertirla en un medio de comunicación comercial.

La presente se fundamenta en un acercamiento a las personas que ya tienen una marca personal y que es utilizada como medio de mercadeo. La novedad del tema de una marca personal gestionada por redes sociales genera que en la mayoría de los entrevistados este procedimiento lo realice de manera empírica; conllevando a que se deba analizar lo realizado por los entrevistados frente a investigaciones anteriores o los aportes que realicen los autores especialistas en el tema. De esta forma, se puede realizar una intersección entre lo que se realiza empíricamente y cómo esto se puede nutrir a partir de lo académico.

\section{Referentes Teóricos}

Para el presente ejercicio, se planteó como objetivo principal identificar el proceso que realizan las personas para la exposición de su marca, usando las redes sociales para tal fin. Para entender y analizar cómo una marca personal es gestionada a través de este medio de comunicación es necesario plantear algunos parámetros que sirvan de ejes conceptuales sobre los cuales apoyar la literatura interpretativa acerca del objetivo. 


\section{Marca}

Primero, se debe tener claro que la marca es lo que identifica al producto, la que hace visible al mismo y por ende ésta le agrega valor al producto o servicio, lo que es percibido por el consumidor del mismo y por el cual está dispuesto a pagar. La marca no es solo identificar o diferenciar al producto, también es cómo los clientes perciben y compran los productos. "Un producto es algo que se elabora en una fábrica, mientras que la marca es aquello que compran los consumidores". (Temporal y Lee 2003), siendo esta un valor estratégico fundamental para la empresa (Fernández, 2013).

Es importante entender que la marca va más allá del producto o servicio -trasciende-, se alimenta del producto original y hasta genera más impacto en el consumidor que el mismo producto o servicio. Es así que la percepción o decisión de compra es guiado por la misma. Una definición cercana a este contexto es: "la personalidad o identidad de un producto, gama de productos o de una organización, derivada de la percepción del consumidor respecto a los atributos" (Arnold, 1993).

La identidad de marca es un conjunto de activos y pasivos vinculados al nombre y símbolo de la misma, en los cuales se incorpora el valor que suministra un producto o servicio a la compañía o a sus clientes (Aaker, 2013). La identidad de una marca es netamente aspiracional, representando lo que la empresa aspira a ser; la razón de su existencia.

\section{Marca personal}

La marca no solo es utilizada por productos o servicios. Países, partidos políticos o personas pueden ser marcas, generando que en la actualidad todo puede ser marca (Rodríguez y Fernández, 2017) hasta las personas pueden ser marcas (Díaz, 2017), generando que todos tengamos el poder de ser nuestra propia marca y nuestro principal trabajo es ser nuestro propio vendedor (Bernal et al , 2020). En el libro Be Your Brand, McNally y Speak (2009) plantean la construcción de una marca para una persona; comienza con la identificación de las características de la persona, de sus dimensiones como marca. A partir de la misma se pueden generar estrategias según su 
planteamiento. Los autores definen a la marca personal como la percepción de la misma asociada a nuestra realidad vital, es el acto de cómo quiere ser recordado (Cortés et al., 2016).

Para Perez (2014) está compuesta por cuatro pilares:

Propósito: la marca para perdurar debe tener un objetivo claro.

Perfil: mostrar lo que transmite, en un entorno de originalidad.

Permanencia: tener un propósito claro y definido.

Profundidad: se define como el nivel de penetración en la mente de la audiencia. Cuantas más veces se muestren las cualidades, y en el mayor número de medios posibles, más profundidad alcanzará y su olvido no será fácil.

La marca personal debe tener atributos relacionados con lo que esta expresa, generando la potencialización de la misma (Xie, 2013), el ejemplo más claro de este fenómeno son los atletas, quienes forjan una marca a partir de sus logros, esfuerzos o historias de superación, convirtiéndolos en una marca que en algunos casos es explotada comercialmente, Hodge y Walker (2015) analizan cómo un atleta puede convertirse en una marca personal, aquella que es apoyada por patrocinadores que buscan apropiarse o aprovecharse del reconocimiento del deportista. Se trata de las estrategias más utilizadas para la creación de la misma, planteando al patrocinio como un estímulo para la creación de una marca personal.

\section{Redes sociales}

Las redes sociales se han convertido en un fenómeno muy influyente en la comunicación de las últimas décadas (Kaplan y Haenlein, 2010). Una de las características más importantes de estos medios sociales son la interacción continua entre las personas, la existencia de convenciones, la voluntad de éstas, en el interactuar y la velocidad con las que se desarrollan las relaciones (Fuchs, 2008), el concepto es ampliado por Bustos y Ruiz (2016) al afirmar que su principal función se basa en la creación de comunidades a partir de usuarios conectados gracias a la flexibilidad que ofrece la tecnología y los bajos costos de la misma, teniendo en cuenta el escenario actual de la comunicación basado en cuatro elementos: hiper conectividad, información social, deslocalización y multi pantalla (Fernández, 2015). 
Por esto la gran importancia de las redes sociales tanto para los individuos como para las empresas, pues fortalecen los lazos sociales existentes, facilitando el desarrollo de una marca personal y que su gestión impacte fácilmente a personas conectadas a dichos medios, a lo anterior se suma el rol de la internet a partir de la masificación de las mismas creando espacios para una comunicación diferente, en la que todos pueden participar por igual, siendo la interacción la clave en los procesos de comunicación que proponen los medios sociales (Moya y Herrera, 2015), compartir intereses genera la creación de redes, y con el planteamiento de comunicación actual, genera que la interacción se facilite con periodistas, políticos o artistas (Lanusse, 2017), facilitando en la exposición de una marca personal, impactando a las personas que la sigan, en este aspecto la exposición de marca se aumenta a partir de la red de contactos del seguidor (Martínez y Lara, 2014)

\section{Redes sociales y marketing}

La necesidad de marcas por lograr la atención del consumidor conlleva al uso de medios no tradicionales de comunicación de marketing, es así como se las redes sociales se constituyen un gran oportunidad para lograr ese acercamiento entre marcas y consumidores (Hudson et al., 2015), conllevando ceder al consumidor cierto grado de control sobre la conversación, permitiendo escuchar al mismo sobre sus experiencias, que nutren a la imagen de marca, permitiendo generar relaciones con las personas y hablar con su mismo lenguaje (Castelló et al., 2016). Las redes sociales constituyen una necesidad dentro de las estrategias empresariales (Real et al., 2014), y como fórmula más utilizada dentro de esta forma de publicidad es el branded content (Castelló et al., 2016), destacándose el video como uno de los medios más efectivos para la transmisión del mensaje el cual es 12 veces más efectivo que cualquier medio publicitario (Afshar, 2017), teniendo en cuenta que la mayoría de búsquedas por los usuarios se realiza en plataformas como YouTube (De Aguilera et al., 2019), pero adicional a este contenido debe contener aspectos que sean valorados por el consumidor, dentro de estos se encuentran: uso de un lenguaje empático, contenido relevante, contenido audiovisual y autenticidad en el mismo (Ruiz, 2017).

El uso de contenidos en la estrategia de marketing, conlleva a el uso de personas desarrolladoras de los mismos, los cuales logran generar una marca personal que impacta y genera la audiencia 
que los sigue o consume sus contenidos (Uribe et al., 2017), lo anterior se constituye en una nueva herramienta de marketing, a partir de la creacion de la marca personal y de un medio por el cual se genera gran exposicion de la misma como son las redes sociales (Fernández et al., 2018), conocidos coloquialmente como influencers, teniendo la capacidad de decision en la compra, que puede superar el control de la marca (Sanz et al, 2019) .

\section{Gestión de marca}

Para llevar a cabo la gestión de una marca se debe recurrir a distintos medios de comunicación, en pro de desarrollar y mantener la conciencia, la identidad y la preferencia por la misma (Paris et al., 2015); un ejemplo, como se mencionó anteriormente, son las redes sociales que facilitan la transmisión del mensaje de la marca a sus consumidores, convirtiéndose en la principal vía para la gestión de publicidad y marca (López et al., 2018). Distintas fuentes académicas y profesionales sostienen que el branded content, como un nuevo enfoque de comunicación de marca consistente en transmitir los mensajes en un contexto de entretenimiento, es la ruta idónea para recuperar la atención de la audiencia (Mourente, 2012). Una tarea complicada para las marcas en la actualidad debido al entorno competitivo en el que se encuentran, radica en que los públicos tienen dificultades para identificar y diferenciar los productos, servicios y organizaciones existentes en un mercado o sector de actividad (Capriotti, 2009).

Todo producto o servicio debe realizar la gestión efectiva de su marca, lo que le permitirá diferenciarse en el mercado y así alcanzar sus objetivos, generando la necesidad de un escenario en el que prime más la estrecha interacción entre marca y audiencias (Sanz et al, 2020). La diferenciación, además de los productos o servicios, es usada por personas, generando la importancia de la marca personal como fenómeno. La revolución de internet y el fácil acceso a la misma también facilita una naciente generación de personas que usan la marca personal. El efecto de una buena gestión, en algunos casos, genera que la persona se convierta en un influenciador. En la actualidad, se encuentran en la red social los Influenciadores de Medios Sociales (IMS), como lo plantea en el estudio $¿$ Who are the social media influencers? A study of public perceptions of personality de Freberg et al. (2011) en el que se analiza cómo son los IMS, sus atributos y cómo son percibidos por sus seguidores, lo anterior facilitando la optimización del capital de los IMS. 
Es interesante conocer qué tienen los IMS o cómo gestionan las redes sociales, que no es más que la gestión de una marca personal.

La importancia en el desarrollo de una marca personal radica en que las personas deben generar impacto en su actuación profesional. Por lo anterior, se hace necesario la exposición de ésta, desarrollando el concepto de branding personal. Las personas y sus carreras se comercializan como marcas completas con promesas de rendimiento, diseños especializados y líneas de identificación para el éxito (Lair et al., 2005). Debido a que la marca personal ofrece una invitación tan sorprendentemente abierta a la autocomplacencia, el fenómeno invita a un análisis cuidadoso y exhaustivo.

\section{Metodología}

De acuerdo con Sampieri (2014) existen tres enfoques de la investigación: el cuantitativo, el mixto y el cualitativo, siendo este último la metodología aplicada a la presente investigación. Como método investigativo, se utilizó el análisis de contenido, siguiendo los lineamientos del autor Krippendorff (1990).

El instrumento que se generó para realizar la presente investigación se basó en una entrevista semiestructurada, en la cual se plantearon diferentes preguntas con temas relacionados a la marca personal, basados en el análisis de contenidos del autor Krippendorff (1990).

Las preguntas planteadas se realizaron de acuerdo a la lectura de los diferentes autores consultados y como resultado, se logró formular 12 preguntas guía con respuestas abiertas para analizar los segmentos entrevistados: cómicos, actores, humoristas, modelos, formadores en coach de liderazgo y presentadores (Tabla 1).

La población objeto de estudio se seleccionó bajo los siguientes criterios de inclusión:

- Que tuvieran una marca personal.

- Que utilizaran las redes sociales para la Exposición de la misma.

- Que tuvieran una cantidad cercana o superior a los 1.000 seguidores en sus redes sociales. 


\section{Tabla 1}

Preguntas realizadas en la entrevista

1 ¿Qué lenguaje utiliza en sus apariciones en redes sociales?

2 ¿Cómo identifica el lenguaje más apropiado para sus contenidos?

3 ¿Al generar sus contenidos para la web, este se alimenta de atributos comunes de su audiencia o responde a una necesidad de transmitir un mensaje?

4 ¿Usted por qué cree que su estilo es atractivo o cercano a sus seguidores?

5 ¿Qué proceso siguió usted para poder identificar que estilo era el más apropiado para incursionar en las redes sociales?

6 ¿Cómo relaciona sus participaciones en la web, con su vida normal o fuera de la web?

7 ¿Cuál es el proceso que realiza para poder medir el impacto de cada una de sus contenidos (videos, fotos, mensajes) entre sus seguidores?

8 ¿Cuál fue el proceso para la generación de su nombre como marca?

9 ¿Cómo identifica qué tipo de seguidores a los que dirigirá sus mensajes?

10 ¿Cuándo inicia su gestión de marca personal en la web, usted trabajo en la segmentación de algún tipo de seguidores en especial?

Cada contenido que usted sube a la web corresponde a una historia o algún acontecimiento actual, ¿estos antes de ser subidos a la web pasan por algún proceso análisis, donde se miren temas que pueden ser vetados o generar algún impacto a su imagen como desarrollador de contenidos?

12 Al ser una imagen de ciertas marcas, estas en algún momento le solicitan no tocar algunos temas, para la protección de ellos como marca comercial, ¿cómo lo maneja?

Fuente: elaboración propia - diseño de entrevista.

Como criterio de exclusión se estableció que tuviera menos de 1.000 seguidores independiente de la red social en la que tuviera presencia.

Como resultado, se seleccionó a un grupo de 15 personas a las que se aplicó la entrevista. Es importante aclarar que la población total que cumple los criterios seleccionados es muy extensa, generando la necesidad de una muestra representativa que abarcara marcas personales que tuvieran un trabajo profesional en su gestión y que realizaran un trabajo empírico en el desarrollo de la 
misma, conllevando a un muestreo por conveniencia, teniendo como resultado las personas relacionadas en la Tabla 2.

\section{Tabla 2}

Descripciones participantes

\begin{tabular}{lcrrrr}
\hline \multirow{2}{*}{ Categoría } & Número & \multicolumn{4}{c}{ Seguidores por red } \\
\cline { 3 - 6 } & entrevista & Facebook & Twitter & Instagram & YouTube \\
\hline Comediante & 1 & 2.100 .000 & 36.000 & 2.000 .000 & 548.000 \\
Presentador & 2 & 1.097 & 387 & & \\
Actor & 3 & 2.500 & 588 & 2.756 & \\
Presentador & 4 & 7.500 .000 & 175.000 & 331.000 & 527 \\
Presentador & 5 & 23.869 & 1.829 & 2.563 & 9.500 \\
Comediante & 6 & 1.031 & & & \\
Comediante & 7 & 603.000 & & & \\
Comediante & 8 & 5.169 & & 38.200 & 199 \\
Liderazgo & 9 & 176 & & 108.000 & 1.383 \\
Comediante & 10 & 530 & 151 & 2.639 & 25 \\
Presentador & 11 & 339 & 3 & 12.700 & 49 \\
Liderazgo & 12 & 64 & 683 & 1.817 & 363 \\
Modelo & 13 & 74 & & 8.689 & \\
Cantante & 14 & 81 & & 63.400 & 431 \\
Modelo & 15 & 1.408 & & 11.000 & \\
\hline
\end{tabular}

Fuente: elaboración propia, base de datos entrevistados.

Los entrevistados fueron agrupados en 6 categorías:

- Comediantes

- Presentadores

- Cantantes

- Actores

- Coach de Liderazgo

- Modelos

Se contactó a las 15 personas que realizarían las entrevistas; la duración estimada por cada entrevista fue de 45 minutos aproximadamente, teniendo en cuenta que algunas se hicieron a través de medios como video o grabación de voz. En algunos casos la duración fue más prolongada según la información aportada por el entrevistado. 
Para el diseño de las preguntas se tuvo en cuenta los siguientes aspectos:

- La frecuencia en el uso de redes sociales utilizando como estadística los seguidores online.

- El tipo de tecnología utilizada (videos editados, caseros, fotos con mensajes, montajes humorísticos, etc.).

- Autenticidad del personaje.

- Gestión de la marca.

- Lenguaje utilizado.

- Mercado objetivo.

Finalmente, luego de recolectar la información, se procedió a tabularla con el uso de la herramienta QDA MINERLITE a fin de identificar códigos y categorías emergentes objeto de análisis en este estudio.

Estos códigos se identificaban al analizar las respuestas a cada pregunta por parte de los entrevistados. El poder identificar palabras clave o frecuentes, permitió volverlas códigos para ser analizados. En la Tabla 3 se pueden observar los códigos generados.

\section{Tabla 3}

Códigos Análisis

\begin{tabular}{llc}
\hline \multicolumn{1}{c}{ Categoría } & \multicolumn{1}{c}{ Código } & Frecuencia \\
\hline Pregunta 5 & Estadísticas & 10 \\
Pregunta 5 & Tecnología & 12 \\
Pregunta 10 & Off Line & 9 \\
Pregunta 9 & La Gente & 12 \\
Pregunta 1 & Autentico & 9 \\
Pregunta 9 & No Hay Proceso & 10 \\
Pregunta 8 & Marca Personal & 6 \\
Pregunta 8 & Segmentación & 8 \\
Pregunta 4 & Naturalidad & 6 \\
Pregunta 3 & Caracterización & 9 \\
Pregunta 11 & Análisis & 8 \\
\hline
\end{tabular}

Fuente: codificaciones software QDA miner lite. 


\section{Análisis de Datos}

Para facilitar el análisis de los procesos de gestión en una marca personal, se tomaron los 11 códigos arrojados por el software QDA MINERLITE y se realizaron 5 agrupaciones principales que sirvieron de referencia para identificar qué aporte u opinión realizaron los entrevistados frente a la misma. En la Tabla 4 se puede observar la agrupación y el peso de cada una.

\section{Tabla 4}

Agrupación Códigos

Fuente: elaboración propia. Entrevista - Análisis de categorías.

\begin{tabular}{lc}
\hline Agrupaciones códigos & Total \\
\hline Sello personal & $37 \%$ \\
Público objetivo & $29 \%$ \\
Lenguaje & $21 \%$ \\
Sentimiento & $7 \%$ \\
Valores & $6 \%$ \\
\hline Total & $100 \%$ \\
\hline
\end{tabular}

\section{Resultados}

La información recolectada en la presente investigación se condenso a partir de la agrupación de códigos anteriormente mostrados, facilitando el análisis de las respuestas de cada entrevistado, guiando la investigación al alcance del objetivo planteado.

A continuación, se observa la condensación de las agrupaciones de códigos y las respuestas de los entrevistados respecto al grupo de preguntas que apuntaban al código agrupado.

\section{Sello personal}

La exposición de un marca personal se constituye en un proceso que involucra la transmisión de un mensaje o un estilo de vida, como se observa en la Tabla 5, los entrevistados afirman que la originalidad es el sello personal de su marca personal, se puede observar que los entrevistados son de profesiones diferentes, pero coinciden en la originalidad de su personaje o marca en las redes sociales como factor vital para poder generar la exposicion de su marca personal. 


\section{Público objetivo}

El sello personal es el factor diferenciador de cada marca, lo anterior se evidencia al analizar los seguidores de cada marca, estos se acercan a cada marca según la conexión que tengan con la misma, al observar las respuestas de los entrevistados afirman identificarlos y por que estos siguen a su marca, dentro de estos testimonios de los entrevistados se encuentran afirmaciones acerca de clasificarlos como personas jovenes, a partir de los contenidos que generan, la marca debe desarrollar contenidos para el publico que identifica como objetivo de la misma.

\section{Valores}

La marca debe ser real y creíble a su mercado objetivo, la exposición de un marca personal se convierte en un gran medio para la promoción de marcas corporativas, más los dueños de la marca personal, no desean perder el terreno trabajado en la construcción de la misma, para lo anterior manfiestan la importancia de la credibilidad del producto asociado a su marca, no pretenden engañar al público, lo que generaría inconvenientes en el desarrollo y exposición negativa de su marca.

\section{Sentimiento}

En este apartado se genera la diferenciación entre una marca corporativa y una personal, la generación de sentimientos entre el público objetivo no es ajeno a las marcas corporativas, pero la respuesta a este es más inmediato en una marca personal, teniendo en cuenta que su construcción parte de una persona para personas, lo anterior facilita que la respuesta a la necesidad de su público objetivo tenga una respuesta más inmediata y de mayor impacto, como se observa en la Tabla 6. Uno de los participantes afirma ajustar sus contenidos a las respuestas de su público frente a sus relaciones amorosas.

\section{Lenguaje}

El desarrollo de contenidos debe ser acorde al público objetivo, esto puede generar que este sea cercano a la marca por la pertinencia que sus publicaciones tienen con este, estas publicaciones en muchas ocasiones son de contenido audiovisual, con la necesidad de entender que el lenguaje debe ser acorde a su mercado objetivo, en la Tabla 6 se observa como algunos desarrolladores generan 
este lenguaje de acuerdo a sus seguidores, pero algunos modifican este según el contenido, el producto tiene una personalidad y por ende el lenguaje del desarrollador de contenidos debe ser acorde a lo que transmita este, de lo contrario la relación entre la marca personal y la del producto no sería exitosa por ende confusa para los consumidores. 


\section{Tabla 5}

Agrupación respuestas según códigos

\begin{tabular}{|c|c|c|c|c|c|}
\hline Categoría & Aportes entrevistados & Categoría & Aportes entrevistados/teoría & Categoría & Aportes entrevistados/teoría \\
\hline $\begin{array}{c}\text { SELLO } \\
\text { PERSONAL }\end{array}$ & $\begin{array}{l}\text { El entrevistado } 11 \text { afirma "Yo } \\
\text { quiero ser más natural, como salen } \\
\text { las cosas; me sería muy difícil ser } \\
\text { un personaje diferente en la red". } \\
\text { El entrevistado } 8 \text { afirma "No } \\
\text { naturalidad, siempre a mis amigos } \\
\text { les ha causado risa mi manera de } \\
\text { ser, yo soy muy imprudente". } \\
\text { Ruiz (2017) relaciona el éxito de } \\
\text { una estrategia de contenidos con la } \\
\text { autenticidad del mismo, el cual es } \\
\text { valorado por los seguidores de la } \\
\text { marca. } \\
\text { Perez (2014) lo define como } \\
\text { mostrar lo que queremos transmitir } \\
\text { en un entorno original. } \\
\text { Arnold (1993) afirma que este es } \\
\text { la personalidad del producto } \\
\text { formada por la percepción del } \\
\text { consumidor. }\end{array}$ & $\begin{array}{l}\text { PÚBLICO } \\
\text { OBJETIVO }\end{array}$ & $\begin{array}{l}\text { El entrevistado } 6 \text { afirma "Yo la } \\
\text { tengo clara, son los comerciantes. } \\
\text { Mis comerciantes son los } \\
\text { informales, ese es mi mercado. } \\
\text { El entrevistado } 15 \text { afirma "No } \\
\text { uso un proceso muy riguroso. Sé } \\
\text { que me siguen mucha gente joven } \\
\text { y es por los contenidos; la verdad } \\
\text { no tengo mucha gente adulta, casi } \\
\text { todos son jóvenes: desde los } 15 \\
\text { hasta los } 25 \text {, que es la gente que } \\
\text { comparte y le gusta lo que hago". } \\
\text { Capriotti (2009) genera la } \\
\text { apreciación del mismo a través de } \\
\text { la necesidad que la marca se } \\
\text { diferencie en el mercado al cual } \\
\text { desea llegar. }\end{array}$ & VALORES & $\begin{array}{l}\text { El entrevistado } 13 \text { afirma "La } \\
\text { verdad es que trato de trabajar con } \\
\text { marcas que me gusten, para no } \\
\text { tener que mentirles a mis } \\
\text { seguidores, yo recomiendo lo que, } \\
\text { o sea las marcas con que trabajo } \\
\text { tienen que ser acorde a mí para yo } \\
\text { poder hacer la mejor publicidad } \\
\text { posible. } \\
\text { El entrevistado } 4 \text { afirma "No me } \\
\text { parece atractiva. Es dejarse uno } \\
\text { manociar y es dejar su trabajo y } \\
\text { dedicación para colocarlo al } \\
\text { servicio de otra marca y desdibuja } \\
\text { el trabajo que hemos hecho". } \\
\text { Aaker (2013) muestra los valores } \\
\text { como un conjunto de activos y } \\
\text { pasivos que son incorporados al } \\
\text { producto. } \\
\text { Xie (2013) afirma que la marca } \\
\text { debe tener atributos relacionados } \\
\text { con lo que expresa, para su } \\
\text { potenciación. }\end{array}$ \\
\hline
\end{tabular}

Fuente: elaboración propia - Resultados investigación. 


\section{Tabla 6}

Agrupación respuestas según códigos

\begin{tabular}{|c|c|c|c|}
\hline Categoría & Aportes entrevistados/teoría & Categoría & Aportes entrevistados/teoría \\
\hline SENTIMIENTO & $\begin{array}{l}\text { El entrevistado } 10 \text { afirma } \\
\text { "Todo caleño es chicanero; yo } \\
\text { también soy chicanero... ve } \\
\text { pues vamos a salir el chicanero, } \\
\text { y de ahí salió el chicanero. } \\
\text { El entrevistado } 7 \text { afirma "Eso } \\
\text { se lo dejo a mi manager: él } \\
\text { toma los datos del Face e } \\
\text { Instagram, mide las cosas } \\
\text { chéveres y las que no tanto para } \\
\text { mejorar. Ya tenemos claro que } \\
\text { las seguidoras mujeres no les } \\
\text { gusta que saque la foto con mi } \\
\text { novia, entonces ya salgo solo". }\end{array}$ & LENGUAJE & $\begin{array}{l}\text { El entrevistado } 1 \text { afirma "Se le } \\
\text { habla a la gente como si fueran } \\
\text { cercanos". } \\
\text { El entrevistado } 9 \text { afirma "Es } \\
\text { un lenguaje que busca un } \\
\text { socialbook, teniendo en cuenta } \\
\text { que deseo generar credibilidad a } \\
\text { mis visitantes. Uso en ocasiones } \\
\text { un lenguaje más científico y } \\
\text { después lo convierto en más } \\
\text { conversacional, como si hablara } \\
\text { con mis seguidores". } \\
\text { Ruiz (2017) el uso de un } \\
\text { lenguaje empático es primordial } \\
\text { para la aceptación del contenido } \\
\text { por parte del público, } \\
\text { generando, aumento en la } \\
\text { exposición de marca. }\end{array}$ \\
\hline
\end{tabular}

Fuente: elaboración propia - Resultados investigación. 


\section{Conclusiones}

En este apartado, se consolidó un acercamiento entre los aportes que realizaron los entrevistados. Lo anterior conllevó a generar recomendaciones frente a las cinco agrupaciones de códigos generadas con el software QDA MINERLITE.

La exposición de la marca personal debe partir de tener una personalidad atractiva para el público, pero ante todo ésta debe partir de algo real, que refleje lo que es la persona. La facilidad de interacción con los seguidores en redes sociales exige que ésta sea real, de lo contrario, su resultado no será el mejor en términos de exposición.

Así como se debe tener claro el público objetivo, el lenguaje a utilizar debe ser cercano al mismo, lo cual debe aplicar para el desarrollo de contenidos, pero debe ser acorde a una personalidad de marca real. Los seguidores piden interacción y por ende la marca debe ser cercana a ellos.

La exposición por redes sociales puede generar un impacto mínimo de 3,5 veces el número de seguidores que tenga la marca. Esta cifra se basa en la conformación de hogares en nuestro país (Minsalud, 2015), teniendo a la familia como grupo primario de influencia. La facilidad de compartir contenidos en redes sociales permite que muchas personas vean y opinen de la marca.

Por lo tanto, es importante cuidar lo que esta transmite, pues se debe generar una administración consciente y responsable de la misma. La marca se debe a sus seguidores y a la red de cada uno: si los contenidos o la imagen de la marca no es administrada pensando en su impacto, esta no crecerá en seguidores y exposición. Las marcas generan su mensaje a partir de la credibilidad de la misma; por ello, dicho mensaje debe ser claro frente a lo que es y representa la marca personal.

La gran diferencia entre una marca corporativa y una marca personal se encuentra en la dimensión de sentimiento, mientras las marcas personales afirman tener está presente en la construcción de la misma y al momento de generar sus contenidos, en los apartados teóricos 
referentes a la gestión de marcas corporativas no se evidencia, lo anterior en un medio de comunicación como las redes sociales juega un papel importante dada su cercanía a sus seguidores.

\section{Referencias}

(1) Aaker, D. (2013). Find the shared interest: A route to community activation and brand building. Journal of Brand Strategy, 2(2), 134-145.

(2) Afshar, V. (2017, 12 de enero). How video marketing will change the game for marketers in 2016. The Huffington Post. https://www.huffpost.com/entry/how-video-marketingwill-_b_8962102

(3) Alonso, A. (2014). Personal Branding: La importancia de la marca personal [Tesis de pregrado, Universidad de Leon].

(4) Arnold, D. (1993). Manual de la Gerencia de Marca. Grupo Norma.

(5) Arques, N. (2012). Y tú, ¿Que marca eres? Alienta.

(6) Bernal, J., Cifuentes, R., \& Garnica, R. (2020). Importancia de la marca personal en la formacion de profesionales de la administracion de empresas. 3C Empresa, $\begin{array}{llll}\text { Investigacion } & y & \text { pensamiento, } & 9(3),\end{array}$ https://doi.org/10.17993/3cemp.2020.090343.79-95

(7) Bustos, J., \& Ruiz, F. (2016). La imagen en twitter como nuevo eje de la comunicacion politica. Opcion, 32(7), 271 - 290.

(8) Capriotti, P. (2009). Branding Corporativo. Fundamentos para la gestión estratégica de la Identidad Corporativa. Coleccion de libros de la empresa.

(9) Castelló, A., Del Pino, C., \& Tur-Viñes, V. (2016). Estrategias de contenido con famosos en marcas dirigidas a publico adolescente. Icono, 14(1), 123-154. https://doi.org/10.7195/ri14.v14i1.883 
(10)Cortés Arévalo, C., Sánchez Sáenz, S., \& Alonso González, A. (2016). La marca personal como elemento decisorio de un buen maestro. Libre Empresa, 13(2), 73-84. https://doi.org/10.18041/libemp.2016.v13n2.26205

(11)De Aguilera, M., Castro, A., \& Pérez, J. (2019). Between broadcast yiourself and broadcast whatever: Youtube's homepage as a synthesisof its bussines strategy. Profesional de la informacion, 28(2). https://doi.org/10.3145/epi.2019.mar.06

(12)Díaz, L. (2017). Soy marca: quiero trabajar con influencers. Profit.

(13)Fernández, A. (2015). Análisis del uso de los medios por las generaciones más jóvenes. El Movimiento 15M y el Umbrella Movement. Profesional de La Información, 24(4), 371-379. https://doi.org/10.3145/epi.2015.jul.03

(14)Fernández Gómez, J. D., Hernández-Santaolalla, V., \& Sanz-Marcos, P. (2018). Influencers, marca personal e ideología política en Twitter. Cuadernos.info, (42), 19-37. https://doi.org/10.7764/cdi.42.1348

(15)Fernández, J. (2013). Principios de estrategia publicitaria y gestión de marcas. Nuevas tendencias de brand management. McGraw Hill.

(16)Freberg, K., Graham, K., McGaughey, K., \& F. L. (2011). Who are the social media influencers? A study of public perceptions of personality. Public Relations Review, 37, 90-92.

(17)Fuchs, C. (2008). Internet and Society, Social Theory in the information Age. Routledge.

(18) Gonçalves, R., Martins, J., \& Rocha, Á. (2016). Internet e redes sociais como instrumentos potenciadores de negócio. RISTI: Revista Ibérica de Sistemas e Tecnologias de Informação, 09-11.

(19)Hodge, C., \& Walker, M. (2015). Personal branding: A perspective from the professional athlete-level-of-Analysis. International Journal of Sport Management and Marketing, 16(1-2), 112 - 131.

(20)Hoyos, R. (2016.). Branding el arte de marcar corazones. Ecoe Ediciones. 
(21)Hudson, S., Huang, L., Roth, S., \& Madden, T. (2015). The influence of social media on consumer-brand relationships: A three-country study of brand perceptions and marketing behaviours. International Journal of Research in Marketing, 33(1), 27-41. https://doi.org/10.1016/j.ijresmar.2015.06.004

(22) Iribarren, J. L., \& Moro, E. (2011). Affinity Paths and information diffusion in social networks. Social Networks, 33, 134-142. https://doi.org/10.1016/j.socnet.2010.11.003

(23)Kaplan, A., \& Haenlein, M. (2010). Users of de world, united! The challenges and opportunities of social media. Business Horizons, 53(1), 59-68. https://doi.org/10.1016/j.bushor.2009.09.003

(24) Krippendorff, K. (1990). Metodología de análisis de contenido: teoría y práctica. Grupo Planeta (GBS).

(25)Lair, D. J., Sullivan, K., \& Cheney, G. (2005). Marketization and the Recasting of the Professional Self: The Rhetoric and Ethics of Personal Branding. Management Communication Quarterly, 18(3), 307-343. https://doi.org/10.1177/0893318904270744

(26)Lanusse, N. (2017). Las redes sociales como impulsor de la Radio Web. QUESTION, 1(53), 428 - 443. https://perio.unlp.edu.ar/ojs/index.php/question/article/view/3792

(27)López, O., Beltrán, C., Morales, R., \& Cavero, O. (2018). Estrategias de marketing digital por medio de redes sociales en el contexto de las PYMES del Ecuador. CienciAméRica, 7(2), 39-56. https://doi.org/10.33210/ca.v7i2.167

(28) Martínez, S., \& Lara, P. (2014). El big data transforma la interpretacion de los medios sociales. Profesional de la informacion, 23(6), 575-581. https://doi.org/10.3145/epi.2014.nov.03

(29)McNally, D., \& Speak, K. D. (2009). Be your own brand: A breakthrough formula for standing out from the crowd. Berrett, Koehler Publishers.

(30) Minsalud. (2015). Encuesta Nacional de Demografía y Salud.

(31) Mourente, F. (2012). El contenido como herramienta eficaz de comunicacionn de marca. Analisis teorico y empirico. Universidad Rey Juan Carlos. 
(32)Moya, M., \& Herrera, S. (2015). Cómo puede contribuir Twitter a una comunicación política más avanzada. Arbor, 191-257. http://dx.doi.org/10.3989/arbor.2015.774n4012

(33)Paris, R. A., Arango, C., Vargas, R., Viana, L., \& Montalvo, L. (2015). Gestion de marca para PYMES. Universidad de Medellín.

(34)Peres, R. M., Muller, E., \& Mahajan, V. (2010). Innovation diffusion and new research directions . International Journal of Research in Marketing, 27, 91-106.

(35)Perez, A. (2014). Marca personal para dummies. Grupo Planeta.

(36) Real, I., Leyva, A., \& Heredia, J. (2014). Uso e impacto de las redes sociales en las estrategias de marketing de las $\mathrm{PyME}^{\prime}$ s. Revista de Investigación Académica Sin Frontera: División de Ciencias Económicas y Sociales, 19(7), 2-24. https://doi.org/10.46589/rdiasf.v0i19.47

(37)Rodríguez, J., \& Fernández, J. (2017). Teoría y estructura de la publicidad. Sintesis.

(38)Ruiz, J. (2017). Millennials y redes sociales: estrategias para una comunicación de marca efectiva. Miguel Hernández Communication Journal, (8), 347-367. https://doi.org/10.21134/mhcj.v0i8.196

(39) Sampieri, R. (2014). Metodología de la Investigación (6ª Ed.). Mc Graw Hill Education.

(40)Sanz, P., Jiménez, G., \& Elias, R. (2019). La incorporacion de la figura del influencer en las campañas publicitarias: Consecuencias para las agencias de publicidad españolas. AdComunica. Revista Científica de Estrategias, Tendencias e Innovación en Comunicación, (18), 63-86. https://raco.cat/index.php/adComunica/article/view/365728

(41)Sanz, P., Pérez, C., \& Velasco, A. (2020). Hacia un cambio en el sector de moda y lujo. del dominio del influencer a la marca: Gucci, Loewe y Margiela. Revista de Comunicacion, 19(2), 263-285. https://doi.org/10.26441/RC19.2-2020-A15

(42)Temporal, P., \& Lee, K. (2003). Branding de alta tecnología. McGraw Hill Interamericana Editores, S.A.

(43) Uribe, R., Buzeta, C. \& Reyes, J. (2017). Personalidad de marca de los partidos políticos en Chile: una mirada desde los ciudadanos más jóvenes. Cuadernos.info, (41), 89-104. 
(44)Xie, Z. (2013). Brand as a person. Economics and Management School, 5(2), 24-29.

Cómo citar este artículo: David, L. (2021). Exposición de marca personal por medio de redes sociales. Tendencias, 22(2), 107-129. https://doi.org/10.22267/rtend.212202.170 